\title{
Convergence of Halley's method for operators with the bounded second Fréchet-derivative in Banach spaces
}

\author{
Ioannis K Argyros ${ }^{1}$, Yeol Je Cho ${ }^{2 *}$ and Hongmin Ren ${ }^{3}$
}

\author{
*Correspondence: yjcho@gnu.ac.kr \\ ${ }^{2}$ Department of Mathematics \\ Education and the RINS, \\ Gyeongsang National University, \\ Jinju, 660-701, Korea \\ Full list of author information is \\ available at the end of the article
}

\begin{abstract}
In this paper, we present a semi-local convergence analysis of Halley's method for approximating a locally unique solution of a nonlinear equation in a Banach space setting, where we assume that the second Fréchet-derivative is bounded. Numerical examples are used to show that the new convergence criteria can provide better information than those provided by the convergence criteria developed earlier.
\end{abstract}

MSC: 65G99; 65J15; 65H10; 47H17; 49M15

Keywords: Halley's method; Banach space; semi-local convergence; Fréchet-derivative; convergence ball

\section{Introduction}

In this paper, we are concerned with the problem of approximating a locally unique solution $x^{\star}$ of the nonlinear equation

$$
F(x)=0,
$$

where $F$ is twice Fréchet-differentiable operator defined on a nonempty open and convex subset of a Banach space $X$ with values in a Banach space $Y$.

Many problems from computational sciences and other disciplines can be brought in a form similar to equation (1.1) using mathematical modeling [1-3]. The solutions of these equations can rarely be found in a closed form. That is why most solution methods for these equations are iterative. The study about convergence matter of iterative procedures is usually based on two types: semi-local and local convergence analysis. The semi-local convergence matter is, based on the information around an initial point, to give conditions ensuring the convergence of the iterative procedure; while the local one is, based on the information around a solution, to find estimates of the radii of convergence balls.

In this paper, we provide a semi-local convergence analysis for Halley's method defined by [4-6]

$$
x_{n+1}=x_{n}-\Gamma_{F}\left(x_{n}\right) F^{\prime}\left(x_{n}\right)^{-1} F\left(x_{n}\right)
$$

for each $n \geq 0$, where

$$
\Gamma_{F}(x)=\left(I-L_{F}(x)\right)^{-1}, \quad L_{F}(x)=\frac{1}{2} F^{\prime}(x)^{-1} F^{\prime \prime}(x) F^{\prime}(x)^{-1} F(x) .
$$

(c) 2013 Argyros et al.; licensee Springer. This is an Open Access article distributed under the terms of the Creative Commons Attribution License (http://creativecommons.org/licenses/by/2.0), which permits unrestricted use, distribution, and reproduction in any medium, provided the original work is properly cited. 
The convergence of Halley's method has a long history and has been studied by many authors ( $c f .[1,2,4-7]$ and the references therein). The most popular conditions for the semi-local convergence of Halley's method are given as follows.

$\left(C_{1}\right)$ There exists $x_{0} \in D$ such that $F^{\prime}\left(x_{0}\right)^{-1} \in L(Y, X)$, the space of bounded linear operator from $Y$ into $X$;

$\left(C_{2}\right)\left\|F^{\prime}\left(x_{0}\right)^{-1} F\left(x_{0}\right)\right\| \leq \eta$ for any $\eta>0$;

$\left(C_{3}\right)\left\|F^{\prime}\left(x_{0}\right)^{-1} F^{\prime \prime}(x)\right\| \leq M$ for each $x$ in $D$ and $M>0$;

$\left(\mathrm{C}_{4}\right)\left\|F^{\prime}\left(x_{0}\right)^{-1}\left[F^{\prime \prime}(x)-F^{\prime \prime}(y)\right]\right\| \leq K\|x-y\|$ for each $x, y \in D$ and $K>0$.

The corresponding sufficient convergence condition $[1,7]$ is given by

$$
\eta \leq \frac{4 K+M^{2}-M \sqrt{M^{2}+2 K}}{3 K\left(M+\sqrt{M^{2}+2 K}\right)} .
$$

There are simple examples to show that $\left(C_{4}\right)$ is not satisfied. As an example, let $X=Y=\mathbb{R}$, $D=[0,+\infty)$ and define $F(x)$ on $D$ by

$$
F(x)=\frac{4}{15} x^{\frac{5}{2}}+x^{2}+x+1 .
$$

Then we have

$$
\left|F^{\prime \prime}(x)-F^{\prime \prime}(y)\right|=|\sqrt{x}-\sqrt{y}|=\frac{|x-y|}{\sqrt{x}+\sqrt{y}} .
$$

Therefore, there is no constant $K$ satisfying $\left(\mathrm{C}_{4}\right)$. Other examples where $\left(\mathrm{C}_{4}\right)$ is not satisfied can be found in [2].

Using the recurrent relations, Equerro and Hernández [7] expanded the applicability of Halley's method by dropping condition $\left(\mathrm{C}_{4}\right)$ and replacing (1.3) by

$$
M \eta<\frac{4-\sqrt{6}}{5} .
$$

In the present study, we show how to expand even further the applicability of Halley's method using $\left(\mathrm{C}_{1}\right),\left(\mathrm{C}_{2}\right),\left(\mathrm{C}_{3}\right)$ and the center-Lipschitz condition:

$\left(C_{5}\right)\left\|F^{\prime}\left(x_{0}\right)^{-1}\left[F^{\prime}(x)-F^{\prime}\left(x_{0}\right)\right]\right\| \leq L\left\|x-x_{0}\right\|$ for each $x \in D$ and $L>0$.

We have that

$$
L \leq M
$$

holds in general and $\frac{M}{L}$ can be arbitrarily large $[2,4]$. The semi-local convergence analysis of Halley's method requires obtaining upper bounds on the norms $\left\|F^{\prime}(x)^{-1} F^{\prime}\left(x_{0}\right)\right\|$. In the literature, $\left(\mathrm{C}_{3}\right)$ is used to obtain

$$
\left\|F^{\prime}(x)^{-1} F^{\prime}\left(x_{0}\right)\right\| \leq \frac{1}{1-M\left\|x-x_{0}\right\|}
$$

for each $x \in D$. However, if we use $\left(C_{5}\right)$ less expensive and tighter if $L<M$ (see (1.5)), the estimate

$$
\left\|F^{\prime}(x)^{-1} F^{\prime}\left(x_{0}\right)\right\| \leq \frac{1}{1-L\left\|x-x_{0}\right\|}
$$


for each $x \in D$ is obtained. This modification can lead to a tighter convergence analysis and weaker sufficient convergence conditions or error bounds or the location of the solution for Halley's method (see numerical examples). The new approach has already led to such advantages in the case of Newton-type methods $[1,2,4-6]$.

We use a different approach than recurrent relations in our semi-local convergence analysis. The paper is organized as follows. Section 2 contains the semi-local convergence of Halley's method, whereas the numerical examples are presented in the concluding section.

\section{Semi-local convergence}

We present the semi-local convergence analysis of Halley's method. We shall use an additional condition.

Define

$$
a=\frac{M \eta}{2}, \quad \eta_{0}=\frac{\eta}{1-a} .
$$

$\left(C_{6}\right)$ Suppose that

$$
\left(L+\frac{M}{2}\right) \eta<1
$$

and there exists

$$
b \in\left(0,1-L \eta_{0}\right)
$$

which is the minimal positive zero of a function $g$ on $\left(0,1-L \eta_{0}\right)$ given by

$$
g(t)=\frac{\frac{a}{1-\frac{L \eta_{0}}{1-t}}+\frac{a}{1-a}}{\left(1-\frac{L \eta_{0}}{1-t}\right)\left(1-\frac{a}{(1-a)\left(1-\frac{L \eta_{0}}{1-t}\right)^{2}}\left(\frac{a}{1-\frac{L \eta_{0}}{1-t}}+\frac{a}{1-a}\right)\right)}-t .
$$

Then we have

$$
b=\frac{\frac{a}{1-\frac{L \eta_{0}}{1-b}}+\frac{a}{1-a}}{\left(1-\frac{L \eta_{0}}{1-b}\right)\left(1-\frac{a}{(1-a)\left(1-\frac{L \eta_{0}}{1-b}\right)^{2}}\left(\frac{a}{1-\frac{L \eta_{0}}{1-b}}+\frac{a}{1-a}\right)\right)} .
$$

Define

$$
R_{0}=\frac{\eta_{0}}{1-b}, \quad \alpha=L R_{0} .
$$

Then we have

$$
\begin{aligned}
& a \in(0,1), \quad \alpha \in(0,1), \\
& \frac{a}{(1-a)(1-\alpha)^{2}}\left(\frac{a}{1-\alpha}+\frac{a}{1-a}\right)=\frac{M^{2} \eta_{0}}{4(1-\alpha)^{2}}\left(\frac{\eta}{1-\alpha}+\eta_{0}\right)<1 .
\end{aligned}
$$

We can rewrite (2.4) as

$$
b=\frac{\frac{M}{2}\left(\frac{\eta}{1-\alpha}+\eta_{0}\right)}{(1-\alpha)\left(1-\frac{M^{2} \eta_{0}}{4(1-\alpha)^{2}}\left(\frac{\eta}{1-\alpha}+\eta_{0}\right)\right)} .
$$

Let us introduce another condition needed in our main theorem. 
$\left(C_{7}\right)$ Suppose that

$$
d=\frac{\frac{M}{2(1-\alpha)} \eta+\frac{M}{2} \eta_{0}}{(1-\alpha)\left(1-\frac{M}{2(1-\alpha)^{2}} \eta\right)}<1 .
$$

We refer to $\left(\mathrm{C}_{1}\right),\left(\mathrm{C}_{2}\right),\left(\mathrm{C}_{3}\right),\left(\mathrm{C}_{5}\right),\left(\mathrm{C}_{6}\right)$ and $\left(\mathrm{C}_{7}\right)$ as the $(\mathrm{C})$ conditions. Let $U(x, R), \bar{U}(x, R)$ stand, respectively, for the open and closed balls in $X$ with center $x$ and radius $R>0$. Then we can show the following semi-local convergence result for Halley's method.

Theorem 2.1 Let $F: D \subset X \rightarrow Y$ be continuously twice Fréchet differentiable, where $X, Y$ are Banach spaces and $D$ is open and convex. Suppose the $(C)$ conditions and $\bar{U}\left(x_{0}, R_{0}\right) \subset D$. Then the Halley sequence $\left\{x_{n}\right\}$ generated by $(1.2)$ is well defined, remains in $U\left(x_{0}, R_{0}\right)$ for all $n \geq 0$ and converges to a unique solution $x^{\star} \in \bar{U}\left(x_{0}, R_{0}\right)$ of the equation $F(x)=0$. Moreover, the following error estimate holds: for each $n \geq 0$,

$$
\left\|x_{n+2}-x_{n+1}\right\| \leq b\left\|x_{n+1}-x_{n}\right\|
$$

Furthermore, if there exists $R^{\star} \geq R_{0}$ such that

$$
\bar{U}\left(x_{0}, R^{\star}\right) \subseteq D
$$

and

$$
\frac{L}{2}\left(R_{0}+R^{\star}\right)<1
$$

then $x^{\star}$ is the only solution of equation $F(x)$ in $\bar{U}\left(x_{0}, R^{\star}\right)$.

Proof We have, using $\left(\mathrm{C}_{1}\right)-\left(\mathrm{C}_{3}\right)$, that

$$
\begin{aligned}
\left\|I-\left(I-L_{F}\left(x_{0}\right)\right)\right\| & =\left\|L_{F}\left(x_{0}\right)\right\|=\frac{1}{2}\left\|F^{\prime}\left(x_{0}\right)^{-1} F^{\prime \prime}\left(x_{0}\right) F^{\prime}\left(x_{0}\right)^{-1} F\left(x_{0}\right)\right\| \\
& \leq \frac{1}{2}\left\|F^{\prime}\left(x_{0}\right)^{-1} F^{\prime \prime}\left(x_{0}\right)\right\|\left\|F^{\prime}\left(x_{0}\right)^{-1} F\left(x_{0}\right)\right\| \\
& \leq \frac{1}{2} M \eta=a<1 .
\end{aligned}
$$

It follows from (2.9) and the Banach lemma on invertible operators $[2,3]$ that $\left(I-L_{F}\left(x_{0}\right)\right)^{-1}$ exists and

$$
\left\|\left(I-L_{F}\left(x_{0}\right)\right)^{-1}\right\| \leq \frac{1}{1-\left\|L_{F}\left(x_{0}\right)\right\|} \leq \frac{1}{1-a} .
$$

Then, by $\left(\mathrm{C}_{2}\right),(1.2)$ and the above estimate, we get

$$
\begin{aligned}
\left\|x_{1}-x_{0}\right\| & =\left\|\left(I-L_{F}\left(x_{0}\right)\right)^{-1} F^{\prime}\left(x_{0}\right)^{-1} F\left(x_{0}\right)\right\| \\
& \leq\left\|\left(I-L_{F}\left(x_{0}\right)\right)^{-1}\right\|\left\|F^{\prime}\left(x_{0}\right)^{-1} F\left(x_{0}\right)\right\| \\
& \leq \frac{\eta}{1-a}=\eta_{0}<R_{0} .
\end{aligned}
$$


We have, by $\left(C_{5}\right)$,

$$
\left\|I-F^{\prime}\left(x_{0}\right)^{-1} F^{\prime}\left(x_{1}\right)\right\| \leq L\left\|x_{1}-x_{0}\right\| \leq L \eta_{0}<L R_{0}=\alpha<1 .
$$

Hence $F^{\prime}\left(x_{1}\right)^{-1}$ exists and

$$
\left\|F^{\prime}\left(x_{1}\right)^{-1} F^{\prime}\left(x_{0}\right)\right\|<\frac{1}{1-\alpha} .
$$

In view of Halley's iteration, we can write

$$
\left[I-L_{F}\left(x_{0}\right)\right]\left(x_{1}-x_{0}\right)+F^{\prime}\left(x_{0}\right)^{-1} F\left(x_{0}\right)=0
$$

or

$$
F\left(x_{0}\right)+F^{\prime}\left(x_{0}\right)\left(x_{1}-x_{0}\right)=\frac{1}{2} F^{\prime \prime}\left(x_{0}\right) F^{\prime}\left(x_{0}\right)^{-1} F\left(x_{0}\right)\left(x_{1}-x_{0}\right)
$$

or

$$
\begin{aligned}
F^{\prime}\left(x_{0}\right)^{-1} F\left(x_{1}\right) \\
=F^{\prime}\left(x_{0}\right)^{-1}\left[F\left(x_{1}\right)-F\left(x_{0}\right)-F^{\prime}\left(x_{0}\right)\left(x_{1}-x_{0}\right)+F\left(x_{0}\right)+F^{\prime}\left(x_{0}\right)\left(x_{1}-x_{0}\right)\right] \\
=\frac{1}{2} F^{\prime}\left(x_{0}\right)^{-1} F^{\prime \prime}\left(x_{0}\right) F^{\prime}\left(x_{0}\right)^{-1} F\left(x_{0}\right)\left(x_{1}-x_{0}\right) \\
\quad+\int_{0}^{1} F^{\prime}\left(x_{0}\right)^{-1} F^{\prime \prime}\left(x_{0}+\theta\left(x_{1}-x_{0}\right)\right)(1-\theta) d \theta\left(x_{1}-x_{0}\right)^{2} .
\end{aligned}
$$

Hence we get

$$
\begin{aligned}
\left\|F^{\prime}\left(x_{0}\right)^{-1} F\left(x_{1}\right)\right\| & \leq \frac{1}{2} M \eta\left\|x_{1}-x_{0}\right\|+\frac{1}{2} M\left\|x_{1}-x_{0}\right\|^{2} \\
& \leq \frac{M}{2(1-\alpha)} \eta\left\|x_{1}-x_{0}\right\|+\frac{1}{2} M\left\|x_{1}-x_{0}\right\|^{2} \\
& \leq \frac{M}{2}\left(\frac{\eta}{1-\alpha}+\eta_{0}\right)\left\|x_{1}-x_{0}\right\| .
\end{aligned}
$$

Moreover, we have

$$
\begin{aligned}
\left\|L_{F}\left(x_{1}\right)\right\| & =\frac{1}{2}\left\|F^{\prime}\left(x_{1}\right)^{-1} F^{\prime}\left(x_{0}\right) F^{\prime}\left(x_{0}\right)^{-1} F^{\prime \prime}\left(x_{1}\right) F^{\prime}\left(x_{1}\right)^{-1} F^{\prime}\left(x_{0}\right) F^{\prime}\left(x_{0}\right)^{-1} F\left(x_{1}\right)\right\| \\
& \leq \frac{1}{2}\left\|F^{\prime}\left(x_{1}\right)^{-1} F^{\prime}\left(x_{0}\right)\right\|^{2}\left\|F^{\prime}\left(x_{0}\right)^{-1} F^{\prime \prime}\left(x_{1}\right)\right\|\left\|F^{\prime}\left(x_{0}\right)^{-1} F\left(x_{1}\right)\right\| \\
& \leq \frac{M}{2(1-\alpha)^{2}}\left(\frac{M}{2(1-\alpha)} \eta\left\|x_{1}-x_{0}\right\|+\frac{1}{2} M\left\|x_{1}-x_{0}\right\|^{2}\right) \\
& \leq \frac{M^{2}\left(\frac{\eta}{1-\alpha}+\eta_{0}\right)\left\|x_{1}-x_{0}\right\|}{4(1-\alpha)^{2}} \\
& \leq \frac{M^{2} \eta_{0}}{4(1-\alpha)^{2}}\left(\frac{\eta}{1-\alpha}+\eta_{0}\right)<1 .
\end{aligned}
$$


Then $\left(I-L_{F}\left(x_{1}\right)\right)^{-1}$ exists and

$$
\begin{aligned}
\left\|\left(I-L_{F}\left(x_{1}\right)\right)^{-1}\right\| & \leq \frac{1}{1-\left\|L_{F}\left(x_{1}\right)\right\|} \leq \frac{1}{1-\frac{M}{2(1-\alpha)^{2}}\left(\frac{M}{2(1-\alpha)} \eta\left\|x_{1}-x_{0}\right\|+\frac{1}{2} M\left\|x_{1}-x_{0}\right\|^{2}\right)} \\
& \leq \frac{1}{1-\frac{M^{2} \eta_{0}}{4(1-\alpha)^{2}}\left(\frac{\eta}{1-\alpha}+\eta_{0}\right)} .
\end{aligned}
$$

So, $x_{2}$ is well defined and, using (1.2), we get

$$
\begin{aligned}
\left\|x_{2}-x_{1}\right\| & \leq \frac{\left\|F^{\prime}\left(x_{1}\right)^{-1} F^{\prime}\left(x_{0}\right)\right\|\left\|F^{\prime}\left(x_{0}\right)^{-1} F\left(x_{1}\right)\right\|}{1-\left\|L_{F}\left(x_{1}\right)\right\|} \\
& \leq \frac{\frac{M}{2(1-\alpha)} \eta\left\|x_{1}-x_{0}\right\|+\frac{1}{2} M\left\|x_{1}-x_{0}\right\|^{2}}{(1-\alpha)\left(1-\frac{M}{2(1-\alpha)^{2}}\left[\frac{M}{2(1-\alpha)} \eta\left\|x_{1}-x_{0}\right\|+\frac{1}{2} M\left\|x_{1}-x_{0}\right\|^{2}\right]\right)} \\
& \leq \frac{\frac{M}{2}\left(\frac{\eta}{1-\alpha}+\eta_{0}\right)}{(1-\alpha)\left(1-\frac{M^{2} \eta_{0}}{4(1-\alpha)^{2}}\left(\frac{\eta}{1-\alpha}+\eta_{0}\right)\right)}\left\|x_{1}-x_{0}\right\|=b\left\|x_{1}-x_{0}\right\| .
\end{aligned}
$$

Therefore, we have

$$
\begin{aligned}
\left\|x_{2}-x_{0}\right\| & \leq\left\|x_{2}-x_{1}\right\|+\left\|x_{1}-x_{0}\right\| \\
& \leq b\left\|x_{1}-x_{0}\right\|+\left\|x_{1}-x_{0}\right\|=(1+b)\left\|x_{1}-x_{0}\right\| \\
& =\frac{1-b^{2}}{1-b}\left\|x_{1}-x_{0}\right\|<\frac{\left\|x_{1}-x_{0}\right\|}{1-b} \leq \frac{\eta_{0}}{1-b}=R_{0} .
\end{aligned}
$$

Hence we have $x_{2} \in U\left(x_{0}, R_{0}\right)$.

The above shows the following items are true for $n=0$ :

(a) $F^{\prime}\left(x_{n+1}\right)^{-1}$ exists and

$$
\left\|F^{\prime}\left(x_{n+1}\right)^{-1} F^{\prime}\left(x_{0}\right)\right\|<\frac{1}{1-\alpha}
$$

(b)

$$
\left\|F^{\prime}\left(x_{0}\right)^{-1} F\left(x_{n+1}\right)\right\| \leq d_{n+1}
$$

(c)

$$
d_{n+1} \leq d d_{n} \leq d^{n+1} d_{0}
$$

(d) $\left(I-L_{F}\left(x_{n+1}\right)\right)^{-1}$ exists and

$$
\left\|\left(I-L_{F}\left(x_{n+1}\right)\right)^{-1}\right\| \leq \frac{1}{1-\frac{M}{2(1-\alpha)^{2}} d_{n+1}} ;
$$

(e) $x_{n+2}$ is well defined and

$$
\left\|x_{n+2}-x_{n+1}\right\| \leq \frac{d_{n+1}}{(1-\alpha)\left(1-\frac{M}{2(1-\alpha)^{2}} d_{n+1}\right)} \leq b\left\|x_{n+1}-x_{n}\right\| \leq b^{n+1}\left\|x_{1}-x_{0}\right\|
$$

(f) $\left\|x_{n+2}-x_{0}\right\|<R_{0}$. 
Here, the sequence $\left\{d_{n}\right\}$ is defined by

$$
\begin{aligned}
& d_{0}=\eta \\
& d_{n+1}=\frac{M}{2(1-\alpha)} d_{n}\left\|x_{n+1}-x_{n}\right\|+\frac{M}{2}\left\|x_{n+1}-x_{n}\right\|^{2}
\end{aligned}
$$

for each $n \geq 0$. The rest will be shown by induction.

Assume that (a)-(f) are true for all natural integers $n \leq k$, where $k \geq 0$ is a fixed integer. Then $F^{\prime}\left(x_{k+2}\right)$ exists since $x_{k+2} \in U\left(x_{0}, R_{0}\right)$ and

$$
\left\|I-F^{\prime}\left(x_{0}\right)^{-1} F^{\prime}\left(x_{k+2}\right)\right\| \leq L\left\|x_{k+2}-x_{0}\right\|<L R_{0}=\alpha<1 .
$$

Hence $F^{\prime}\left(x_{k+2}\right)^{-1}$ exists and

$$
\left\|F^{\prime}\left(x_{k+2}\right)^{-1} F^{\prime}\left(x_{0}\right)\right\| \leq \frac{1}{1-L\left\|x_{k+2}-x_{0}\right\|}<\frac{1}{1-\alpha} .
$$

Next, we estimate $\left\|F^{\prime}\left(x_{0}\right)^{-1} F\left(x_{k+2}\right)\right\|$. It follows from Halley's method that

$$
\begin{aligned}
F\left(x_{k+2}\right)= & F\left(x_{k+2}\right)-F\left(x_{k+1}\right)-F^{\prime}\left(x_{k+1}\right)\left(x_{k+2}-x_{k+1}\right) \\
& +\frac{1}{2} F^{\prime \prime}\left(x_{k+1}\right) F^{\prime}\left(x_{k+1}\right)^{-1} F\left(x_{k+1}\right)\left(x_{k+2}-x_{k+1}\right)
\end{aligned}
$$

or

$$
\begin{aligned}
F^{\prime}\left(x_{0}\right)^{-1} F\left(x_{k+2}\right)= & \frac{1}{2} F^{\prime}\left(x_{0}\right)^{-1} F^{\prime \prime}\left(x_{k+1}\right) F^{\prime}\left(x_{k+1}\right)^{-1} F^{\prime}\left(x_{0}\right) F^{\prime}\left(x_{0}\right)^{-1} F\left(x_{k+1}\right)\left(x_{k+2}-x_{k+1}\right) \\
& +\int_{0}^{1} F^{\prime}\left(x_{0}\right)^{-1} F^{\prime \prime}\left(x_{k+1}+\theta\left(x_{k+2}-x_{k+1}\right)\right)(1-\theta) d \theta\left(x_{k+2}-x_{k+1}\right)^{2} .
\end{aligned}
$$

Hence we get

$$
\begin{aligned}
\left\|F^{\prime}\left(x_{0}\right)^{-1} F\left(x_{k+2}\right)\right\| & \leq \frac{M}{2(1-\alpha)} d_{k+1}\left\|x_{k+2}-x_{k+1}\right\|+\frac{M}{2}\left\|x_{k+2}-x_{k+1}\right\|^{2}=d_{k+2} \\
& =\frac{M}{2}\left(\frac{d_{k+1}}{1-\alpha}+\left\|x_{k+2}-x_{k+1}\right\|\right)\left\|x_{k+2}-x_{k+1}\right\| \\
& \leq \frac{M}{2}\left(\frac{d^{k+1} \eta}{1-\alpha}+b^{k+1}\left\|x_{1}-x_{0}\right\|\right)\left\|x_{k+2}-x_{k+1}\right\| \\
& \leq \frac{M}{2}\left(\frac{d \eta}{1-\alpha}+b \eta_{0}\right)\left\|x_{k+2}-x_{k+1}\right\| \\
& \leq \frac{M}{2}\left(\frac{d \eta}{1-\alpha}+b \eta_{0}\right) b^{k+1}\left\|x_{1}-x_{0}\right\| \\
& \leq \frac{M}{2}\left(\frac{d \eta}{1-\alpha}+b \eta_{0}\right) b \eta_{0}, \\
d_{k+2} \leq \frac{M}{2}\left(\frac{d \eta}{1-\alpha}\right. & \left.+b \eta_{0}\right)\left\|x_{k+2}-x_{k+1}\right\| \\
\leq \frac{M}{2}\left(\frac{d \eta}{1-\alpha}\right. & \left.+b \eta_{0}\right) \frac{d_{k+1}}{(1-\alpha)\left(1-\frac{M}{2(1-\alpha)^{2}} d^{k+1} \eta\right)}
\end{aligned}
$$




$$
\begin{aligned}
& \leq \frac{M}{2}\left(\frac{d \eta}{1-\alpha}+b \eta_{0}\right) \frac{d_{k+1}}{(1-\alpha)\left(1-\frac{M}{2(1-\alpha)^{2}} d \eta\right)} \\
& \leq \frac{M}{2}\left(\frac{\eta}{1-\alpha}+\eta_{0}\right) \frac{d_{k+1}}{(1-\alpha)\left(1-\frac{M}{2(1-\alpha)^{2}} \eta\right)} \\
& =d d_{k+1} \leq d^{k+2} d_{0}
\end{aligned}
$$

and

$$
\begin{aligned}
\left\|L_{F}\left(x_{k+2}\right)\right\| & \leq \frac{1}{2}\left\|F^{\prime}\left(x_{k+2}\right)^{-1} F^{\prime}\left(x_{0}\right)\right\|^{2}\left\|F^{\prime}\left(x_{0}\right)^{-1} F\left(x_{k+2}\right)\right\|\left\|F^{\prime}\left(x_{0}\right)^{-1} F^{\prime \prime}\left(x_{k+2}\right)\right\| \\
& \leq \frac{M}{2(1-\alpha)^{2}} d_{k+2} \leq \frac{M^{2}}{4(1-\alpha)^{2}}\left(\frac{\eta}{1-\alpha}+\eta_{0}\right) \eta_{0}<1 .
\end{aligned}
$$

Thus $\left(I-L_{F}\left(x_{k+2}\right)\right)^{-1}$ exists and

$$
\left\|\left(I-L_{F}\left(x_{k+2}\right)\right)^{-1}\right\| \leq \frac{1}{1-\frac{M}{2(1-\alpha)^{2}} d_{k+2}} \leq \frac{1}{1-\frac{M^{2}}{4(1-\alpha)^{2}}\left(\frac{\eta}{1-\alpha}+\eta_{0}\right) \eta_{0}} .
$$

Therefore, $x_{k+3}$ is well defined. Moreover, we obtain

$$
\begin{aligned}
\left\|x_{k+3}-x_{k+2}\right\| & \leq\left\|\left(I-L_{F}\left(x_{k+2}\right)\right)^{-1}\right\|\left\|F^{\prime}\left(x_{k+2}\right)^{-1} F^{\prime}\left(x_{0}\right)\right\|\left\|F^{\prime}\left(x_{0}\right)^{-1} F\left(x_{k+2}\right)\right\| \\
& \leq \frac{d_{k+2}}{(1-\alpha)\left(1-\frac{M}{2(1-\alpha)^{2}} d_{k+2}\right)} \leq \frac{\frac{M}{2}\left(\frac{\eta}{1-\alpha}+\eta_{0}\right)\left\|x_{k+2}-x_{k+1}\right\|}{(1-\alpha)\left(1-\frac{M^{2}}{4(1-\alpha)^{2}}\left(\frac{\eta}{1-\alpha}+\eta_{0}\right) \eta_{0}\right)} \\
& =b\left\|x_{k+2}-x_{k+1}\right\| \leq b^{k+2}\left\|x_{1}-x_{0}\right\| .
\end{aligned}
$$

Furthermore, we have

$$
\begin{aligned}
\left\|x_{k+3}-x_{0}\right\| & \leq\left\|x_{k+3}-x_{k+2}\right\|+\left\|x_{k+2}-x_{k+1}\right\|+\cdots+\left\|x_{1}-x_{0}\right\| \\
& \leq\left(b^{k+2}+b^{k+1}+\cdots+1\right)\left\|x_{1}-x_{0}\right\| \\
& =\frac{1-b^{k+3}}{1-b}\left\|x_{1}-x_{0}\right\|<\frac{\eta_{0}}{1-b}=R_{0} .
\end{aligned}
$$

Hence we deduce that $x_{k+3} \in U\left(x_{0}, R_{0}\right)$, which completes the induction for (a)-(f).

Let $m$ be a natural integer. Then we have

$$
\begin{aligned}
\left\|x_{k+m}-x_{k}\right\| & \leq\left\|x_{k+m}-x_{k+m-1}\right\|+\left\|x_{k+m-1}-x_{k+m-2}\right\|+\cdots+\left\|x_{k+1}-x_{k}\right\| \\
& \leq\left(b^{m-1}+\cdots+b+1\right)\left\|x_{k+1}-x_{k}\right\| \\
& \leq \frac{1-b^{m}}{1-b} b^{k}\left\|x_{1}-x_{0}\right\| .
\end{aligned}
$$

It follows that $\left\{x_{k}\right\}$ is a complete sequence in a Banach space $X$ and as such it converges to some $x^{\star} \in \bar{U}\left(x_{0}, R_{0}\right)$ (since $\bar{U}\left(x_{0}, R_{0}\right)$ is a closed set). By letting $k \rightarrow \infty$ in (2.10), we obtain $F\left(x^{\star}\right)=0$. We also have

$$
\left\|x^{\star}-x_{k}\right\| \leq \frac{b^{k}}{1-b}\left\|x_{1}-x_{0}\right\| .
$$


To show the uniqueness part, let $y^{\star}$ be a solution of the equation $F(x)=0$ in $\bar{U}\left(x_{0}, R^{\star}\right)$. Let $T=\int_{0}^{1} F^{\prime}\left(x_{0}\right)^{-1} F^{\prime}\left(x^{\star}+\theta\left(y^{\star}-x^{\star}\right)\right) d \theta$. Using $\left(C_{5}\right)$, we have in turn that

$$
\begin{aligned}
\|I-T\| & =\left\|\int_{0}^{1} F^{\prime}\left(x_{0}\right)^{-1}\left[F^{\prime}\left(x^{\star}+\theta\left(y^{\star}-x^{\star}\right)\right)-F^{\prime}\left(x_{0}\right)\right] d \theta\right\| \\
& \leq L \int_{0}^{1}\left[(1-\theta)\left\|x^{\star}-x_{0}\right\|+\theta\left\|y^{\star}-x_{0}\right\|\right] d \theta \\
& \leq \frac{L}{2}\left(R_{0}+R^{\star}\right)<1 .
\end{aligned}
$$

It follows from the Banach lemma on invertible operators [2-4] that $T^{-1} \in L(Y, X)$. Using the identity

$$
0=F^{\prime}\left(x_{0}\right)^{-1}\left(F\left(y^{\star}\right)-F\left(x^{\star}\right)\right)=F^{\prime}\left(x_{0}\right)^{-1} T\left(y^{\star}-x^{\star}\right),
$$

we deduce $y^{\star}=x^{\star}$. This completes the proof.

\section{Numerical examples}

In this section, we give some examples to show the application of our theorem.

Example 3.1 Let us define a scalar function $F(x)=x^{3}-0.64$ on $D=(0,1)$ with initial point $x_{0}=0.85$. Then we have that

$$
F^{\prime}(x)=3 x^{2}, \quad F^{\prime \prime}(x)=6 x .
$$

So, $F\left(x_{0}\right)=-0.025875, F^{\prime}\left(x_{0}\right)=2.1675$ and $\eta=0.011937716$. Moreover, we have, for any $x \in D$,

$$
\left|F^{\prime}\left(x_{0}\right)^{-1} F^{\prime \prime}(x)\right|=\frac{2|x|}{x_{0}^{2}} \leq \frac{2}{x_{0}^{2}}=2.76816609
$$

and

$$
\left|F^{\prime}\left(x_{0}\right)^{-1}\left[F^{\prime}(x)-F^{\prime}\left(x_{0}\right)\right]\right|=\frac{\left|x+x_{0}\right|}{x_{0}^{2}}\left|x-x_{0}\right| \leq 2.560553633\left|x-x_{0}\right| .
$$

That is, we can choose $M=2.76816609$ and $L=2.560553633$ in conditions $\left(C_{3}\right)$ and $\left(C_{5}\right)$, respectively. Hence, we obtain $a=0.016522791, \eta_{0}=0.012138274$ and $1-L \eta_{0}=$ 0.968919297 . Furthermore, it is easy to get $b=0.035021656$ by using iterative methods such as the secant method. Then we have $R_{0}=0.012578805, \alpha=0.032208705$ and $d=0.035628901$. So, conditions $\left(\mathrm{C}_{6}\right)$ and $\left(\mathrm{C}_{7}\right)$ are satisfied. It is clear that

$$
\bar{U}\left(x_{0}, R_{0}\right)=[0.837421195,0.862578805] \subset D .
$$

Now, all the conditions in Theorem 2.1 are true and Theorem 2.1 applies. We can compare our results to the ones in [7]. Using (1.4), we get

$$
\alpha_{0}=M \eta=0.03304558<\frac{4-\sqrt{6}}{5} .
$$


So, the conditions of [7] are also satisfied. The uniqueness ball is $\bar{U}\left(x_{0}, r_{0} \eta\right)$, where

$$
r_{0}=\frac{2-3 \alpha_{0}}{2\left(1-3 \alpha_{0}+\alpha_{0}^{2}\right)}=1.053745860 .
$$

Then we get $R_{1}=r_{0} \eta=0.012579319>R_{0}$. That is, we provide better information on the location of the solution. Moreover, by (2.7) and (2.8), we can set $R^{\star}=0.15$, which extends the uniqueness ball from $\bar{U}\left(x_{0}, R_{0}\right)$ to $\bar{U}\left(x_{0}, R^{\star}\right)$.

Example 3.2 In this example, we provide an application of our results to a special nonlinear Hammerstein integral equation of the second kind. Consider the integral equation

$$
x(s)=1+\frac{4}{5} \int_{0}^{1} G(s, t) x(t)^{3} d t
$$

for any $s \in[0,1]$, where $G$ is the Green kernel on $[0,1] \times[0,1]$ defined by

$$
G(s, t)= \begin{cases}t(1-s), & t \leq s \\ s(1-t), & s \leq t\end{cases}
$$

Let $X=Y=C[0,1]$ and $D$ be a suitable open convex subset of $X_{1}:=\{x \in X: x(s)>0, s \in$ $[0,1]\}$, which is given below. Define a mapping $F: D \rightarrow Y$ by

$$
[F(x)](s)=x(s)-1-\frac{4}{5} \int_{0}^{1} G(s, t) x(t)^{3} d t
$$

for any $s \in[0,1]$. The first and second derivatives of $F$ are given by

$$
\left[F^{\prime}(x) y\right](s)=y(s)-\frac{12}{5} \int_{0}^{1} G(s, t) x(t)^{2} y(t) d t
$$

for any $s \in[0,1]$ and

$$
\left[F^{\prime \prime}(x) y z\right](s)=-\frac{24}{5} \int_{0}^{1} G(s, t) x(t) y(t) z(t) d t
$$

for any $s \in[0,1]$, respectively.

We use the max-norm. Let $x_{0}(s)=1$ for all $s \in[0,1]$. Then, for any $y \in D$, we have

$$
\left[\left(I-F^{\prime}\left(x_{0}\right)\right) y\right](s)=\frac{12}{5} \int_{0}^{1} G(s, t) y(t) d t
$$

for any $s \in[0,1]$, which means

$$
\left\|I-F^{\prime}\left(x_{0}\right)\right\| \leq \frac{12}{5} \max _{s \in[0,1]} \int_{0}^{1} G(s, t) d t=\frac{12}{5 \times 8}=\frac{3}{10}<1 .
$$

It follows from the Banach theorem that $F^{\prime}\left(x_{0}\right)^{-1}$ exists and

$$
\left\|F^{\prime}\left(x_{0}\right)^{-1}\right\| \leq \frac{1}{1-\frac{3}{10}}=\frac{10}{7} .
$$


On the other hand, it follows from (3.6) that $\left\|F\left(x_{0}\right)\right\|=\frac{4}{5} \max _{s \in[0,1]} \int_{0}^{1} G(s, t) d t=\frac{1}{10}$. Then we get $\eta=\frac{1}{7}$.

Note that $F^{\prime \prime}(x)$ is not bounded in $X$ or its subset $X_{1}$. Take into account that a solution $x^{\star}$ of equation (1.1) with $F$ given by (3.6) must satisfy

$$
\left\|x^{\star}\right\|-1-\frac{1}{10}\left\|x^{\star}\right\|^{3} \leq 0
$$

i.e., $\left\|x^{\star}\right\| \leq \rho_{1}=1.153467305$ and $\left\|x^{\star}\right\| \geq \rho_{2}=2.423622140$, where $\rho_{1}$ and $\rho_{2}$ are the positive roots of the real equation $z-1-\frac{1}{10} z^{3}=0$. Consequently, if we look for a solution such that $x^{\star}<\rho_{1} \in X_{1}$, we can consider $D=\left\{x: x \in X_{1}\right.$ and $\left.\|x\|<r\right\}$, with $r \in\left(\rho_{1}, \rho_{2}\right)$, as a nonempty open convex subset of $X$. For example, choose $r=1.7$.

Using (3.7) and (3.8), we have, for any $x, y, z \in D$,

$$
\begin{aligned}
\left|\left[\left(F^{\prime}(x)-F^{\prime}\left(x_{0}\right)\right) y\right](s)\right| & =\frac{12}{5}\left|\int_{0}^{1} G(s, t)\left(x(t)^{2}-x_{0}(t)^{2}\right) y(t) d t\right| \\
& \leq \frac{12}{5} \int_{0}^{1} G(s, t)\left|x(t)+x_{0}(t)\right|\left|x(t)-x_{0}(t)\right| y(t) d t \\
& \leq \frac{12}{5} \int_{0}^{1} G(s, t)(r+1)\left|x(t)-x_{0}(t)\right| y(t) d t, \quad s \in[0,1]
\end{aligned}
$$

and

$$
\left|\left[F^{\prime \prime}(x) y z\right](s)\right|=\frac{24}{5} \int_{0}^{1} G(s, t) x(t) y(t) z(t) d t, \quad s \in[0,1] .
$$

Then we get

$$
\left\|F^{\prime}(x)-F^{\prime}\left(x_{0}\right)\right\| \leq \frac{12}{5} \times \frac{1}{8}(r+1)\left\|x-x_{0}\right\|=\frac{81}{100}\left\|x-x_{0}\right\|
$$

and

$$
\left\|F^{\prime \prime}(x)\right\| \leq \frac{24}{5} \times \frac{r}{8}=\frac{51}{50} .
$$

Now, we can choose constants in Theorem 2.1 as follows:

$$
\begin{array}{ll}
M=\frac{51}{35}, \quad L=\frac{81}{70}, & a=\frac{51}{490}, \quad \eta_{0}=\frac{70}{439}, \\
\left(L+\frac{M}{2}\right) \eta=\frac{66}{245}, & 1-L \eta_{0}=\frac{358}{439}, \\
b=0.4266517573, & R_{0}=0.2781089940 \\
\alpha=0.3218118359, & d=0.5138822165 .
\end{array}
$$

We also have

$$
\bar{U}\left(x_{0}, R_{0}\right) \subset D .
$$


So, all the conditions in Theorem 2.1 are satisfied and Theorem 2.1 applies. Consequently, the sequence $\left\{x_{n}\right\}$ generated by Halley's method (1.2) with initial point $x_{0}$ converges to the unique solution $x^{\star}$ of equation (1.1) on $\bar{U}\left(x_{0}, R_{0}\right)$. Moreover, by (2.7) and (2.8), we can set $R^{\star}=0.7$, which extends the uniqueness ball from $\bar{U}\left(x_{0}, R_{0}\right)$ to $\bar{U}\left(x_{0}, R^{\star}\right)$.

\section{Competing interests}

The authors declare that they have no competing interests.

\section{Authors' contributions}

All authors read and approved the final manuscript.

\section{Author details}

${ }^{1}$ Department of Mathematical Sciences, Cameron University, Lawton, OK 73505, USA. ${ }^{2}$ Department of Mathematics Education and the RINS, Gyeongsang National University, Jinju, 660-701, Korea. ${ }^{3}$ College of Information and Engineering, Hangzhou Polytechnic, Hangzhou, Zhejiang 311402, P.R. China.

\section{Acknowledgements}

First, the authors would like to thank the anonymous referee for valuable suggestions that have been implemented in the final version of this paper. The second author was supported by the Basic Science Research Program through the National Research Foundation of Korea (NRF) funded by the Ministry of Education, Science and Technology (Grant Number: 2012-0008170) and the third author was supported by the National Natural Science Foundation of China (Grant No. 10871178)

Received: 29 November 2012 Accepted: 4 May 2013 Published: 23 May 2013

\section{References}

1. Argyros, IK: The convergence of Halley-Chebyshev type method under Newton-Kantorovich hypotheses. Appl. Math. Lett. 6, 71-74 (1993)

2. Argyros, IK: Computational Theory of Iterative Methods. Studies in Computational Mathematics, vol. 15 Chui, CK, Wuytack L (eds.). Elsevier, New York (2007)

3. Deuflhard, P: Newton Methods for Nonlinear Problems: Affine Invariance and Adaptive Algorithms. Springer, Berlin (2004)

4. Argyros, IK, Cho, YJ, Hilout, S: On the semilocal convergence of the Halley method using recurrent functions. J. Appl. Math. Comput. 37, 221-246 (2011)

5. Argyros, IK, Ren, HM: Ball convergence theorems for Halley's method in Banach spaces. J. Appl. Math. Comput. 38, 453-465 (2012)

6. Argyros, IK, Ren, HM: On the Halley method in Banach space. Appl. Math. (2012, to appear)

7. Ezquerro, JA, Hernández, MA: New Kantorovich-type conditions for Halley's method. Appl. Numer. Anal. Comput. Math. 2, 70-77 (2005)

\section{Submit your manuscript to a SpringerOpen ${ }^{\ominus}$ journal and benefit from:}

- Convenient online submission

Rigorous peer review

- Immediate publication on acceptance

- Open access: articles freely available online

- High visibility within the field

- Retaining the copyright to your article 